\title{
REALCOMPACTIFICATION AND REPLETENESS \\ OF WALLMAN SPACES
}

JAMES CAMACHO, JR.

Jersey City State College

2039 Kennedy Boulevard

Jersey City, New Jersey 07305

U.S.A.

(Received August 20, 1991 and in revised form January 23, 1992)

ABSTRACT. The extension of bounded lattice continuous functions on an arbitrary set $x$ to the set of lattice regular zero-one measures on an algebra generated by a lattice (a Wallman-type space) is investigated.

Next the subset of lattice regular zero-one measures on an algebra generated by a lattice which integrates all lattice continuous functions on $X$ is introduced and various properties of it are presented.

Finally conditions are established using repleteness criteria whereby the space of lattice regular zero-one measures on an algebra generated by a lattice which are countably additive (a Wallmantype space) is realcompact.

KEY WORDS AND PHRASES. Realcompact, repleteness, Wallman spaces, normal lattice, lattice continuous functions.

1991 AMS SUBJECT CLASSIFICATION CODES. 28C15, 28A12, 54D60.

\section{INTRODUCTION.}

Let $X$ be an arbitrary set and $\underline{L}$ a lattice of subsets of $X . A(\underline{L})$ denotes the algebra generated by $\underline{L}$, and $M(\underline{L})$ those bounded finitely additive measures on $A(\underline{L})$, and $M_{R}(\underline{L})$ those elements of $M(\underline{L})$ which are $\underline{L}$-regular while $M_{R}{ }^{\sigma}(\underline{L})$ denotes those elements of $M_{R}(\underline{L})$ which are countably additive. The zero-one valued members of the above are designated by $I(\underline{L}), I_{R}(\underline{L})$, and $I_{R}{ }^{\sigma}(\underline{L})$ respectively. For $A \in A(\underline{L}), w(A)=\left\{u \in I_{R}(\underline{L}) \mid u(A)=1\right\}, w(\underline{L})=\{u(L) \mid L \in \underline{L})$, then $I_{R}(\underline{L})$ with the topology of closed sets $\tau w(\underline{L})$ of arbitrary intersections of sets of $w(\underline{L})$ is a compact, $T_{1}$ topological space. It is one of the Wallman type spaces. Assuming $\underline{L}$ is disjunctive then it is $T_{2}$ if and only if $\underline{L}$ is normal.

We begin by considering briefly, because of their importance, certain fundamental properties of normal lattices. Then we proceed to a consideration of $I_{R}(\underline{L})$, and the extension of bounded lattices continuous functions on $X$ to $I_{R}(\underline{L})$. These results are generally known (see [8]) but we give somewhat shorter more direct proofs here.

We next consider the space $Q(\underline{L})$ of measures in $I_{R}(\underline{L})$ which integrate all lattice continuous functions on $X$, and show its relationship to $I_{R}{ }^{\sigma}(\underline{L})$, and under suitable conditions, its relationship to the $G_{\delta}$-closure of $X$ in $I_{R}(\underline{L})$. 
Finally, we consider the Wallman type space $I_{R}{ }^{\sigma}(\underline{L})$, and the lattice $w_{\sigma}(\underline{L})$, where for $A \in A(\underline{L})$, $w_{\sigma}(A)=\left\{u \in I_{R}{ }^{\sigma}(\underline{L}) \mid u(A)=1\right\}$, and where $w_{\sigma}(\underline{L})=\left\{w_{\sigma}(L) \mid L \in \underline{L}\right\}$. It is well-known that if $\underline{L}$ is disjunctive then $w_{\sigma}(\underline{L})$ is replete. We consider in this space the lattice of closed sets $\tau w_{\sigma}(\underline{L})$ and its associated lattice of zero sets, and investigate their repleteness - thus obtaining sufficient conditions for the space $I_{R}{ }^{\sigma}(\underline{L})$ to be realcompact.

Our notations and terminology is consistent with $[1,3,5,6,11]$. However, the main definitions and notations used throughout the paper are presented for the reader's convenience in section $2(\mathrm{a})$. We note also that a number of results on normal lattices in section 2(b) are related to work of $[4$, 9].

\section{2.(a)BACKGROUND AND NOTATION.}

Let $X$ be an abstract set, and $\underline{L}$ the lattice of subsets of $X$. We assume that $\phi, X \in \underline{L}$ for most of our results. First:

\section{Lattice Terminology:}

$A(\underline{L})$ is the algebra generated by $\underline{L}$.

$\sigma(\underline{L})$ is the $\sigma$-algebra generated by $\underline{L}$.

$\delta(\underline{L})$ is the lattice of all countable intersections of sets from $\underline{L} . \underline{L}$ is a delta lattice ( $\delta$-lattice) if $\delta(\underline{L})=\underline{L}$.

$\tau(\underline{L})$ is the lattice of arbitrary intersections of sets of $\underline{L}$.

$\underline{L}$ is complemented if $L \in \underline{L}=>L^{\prime} \in \underline{L}$ (prime denotes complement), that is, $\underline{L}$ is an algebra.

$\underline{L}$ is separating, if for any two elements $x \neq y$ of $X$, there exists an element $L \in \underline{L}$ such that $x \in L$ and $y \notin L$.

$\underline{L}$ is $T_{2}$ if, for any two elements $x \neq y$ of $X$, there exists $A, B \in \underline{L}$ such that $X \in A^{\prime}$ and $y \in B^{\prime}$ and $A^{\prime} \cap B^{\prime}=\phi$.

$\underline{L}$ is disjunctive if for any $x \in X$ and $A \in \underline{L}$ such that $x \notin A$, there exists a $B \in \underline{L}$ such that $x \in B$ and $A \cap B=\phi$.

$\underline{L}$ is regular if for any $x \in X$, and $A \in \underline{L}$ such that $x \notin A$ there exist $B, C \in \underline{L}$ such that $x \in B^{\prime}$, $A \subset C^{\prime}$ and $B^{\prime} \cap C^{\prime}=\phi$.

$\underline{L}$ is normal if for all $L_{1}, L_{2} \in \underline{L}$ such that $L_{1} \cap L_{2}=\phi$ there exists $\tilde{L}_{1}^{\prime}, \tilde{L}_{2} \in \underline{L}$ such that $L_{1} \subset \tilde{L}_{1}^{\prime}$, $L_{2} \subset \tilde{\tilde{L}}_{2}^{\prime}$, and $\tilde{L}_{1}^{\prime} \cap \tilde{L}_{2}^{\prime}=\phi$.

$\underline{L}$ is compact if every covering of $X$ by elements of $\underline{L}^{\prime}$ has a finite subcovering.

$\underline{L}$ is countably compact if every countable covering of $X$ by .elements of $\underline{L}^{\prime}$ has a finite subcovering.

$\underline{L}$ is Lindelöf if every covering of $X$ by elements of $\underline{L}^{\prime}$ has a countable subcovering.

$\underline{L}$ is countably paracompact if whenever $A_{n} \downarrow \phi, A_{n} \in \underline{L}$ there exists $B_{n} \in \underline{L}$ such that $A_{n} \subset B_{n}^{\prime}$ and $B_{n}^{\prime} \downarrow \phi$.

$\underline{L}$ is complement generated if, for $L \in \underline{L}$ there exists $L_{n} \in \underline{L}$ such that $L=\bigcap_{n}^{\infty} L_{1} L_{n}^{\prime}$.

It is well known that if $\underline{L}$ is complement generated then $\underline{L}$ is countably paracompact.

Measure Terminology

We denote by $M(\underline{L})$ the finitely additive bounded measures on $A(\underline{L})$ (we may and do assume all elements of $M(\underline{L})$ are $\geq 0)$. 
$u \in M(\underline{L})$ is $\underline{L}$-regular if for any $A \in A(\underline{L}), u(A)=\sup \{u(L) \mid L \subset A, L \in \underline{L}\}$; (equivalently) = inf $\left\{u\left(L^{\prime}\right) \mid A \subset L^{\prime}, L \in \underline{L}\right\}$.

$u \in M(\underline{L})$ is $\sigma$-smooth on $\underline{L}$ if $L_{n} \in \underline{L}, n=1,2, \ldots$ and $L_{n} \downarrow \phi=>u\left(L_{n}\right) \rightarrow 0$

$u \in M(\underline{L})$ is $\sigma$-smooth on $A(\underline{L})$ if $A_{n} \in A(\underline{L}), n=1,2, \ldots$ and $A_{n} \downarrow \phi=>u\left(A_{n}\right) \rightarrow 0$. Note $u$ is $\sigma$ smooth on $A(\underline{L})$ iff $u$ is countably additive.

We will use the following notations.

$M_{R}(\underline{L})=$ the set of $\underline{L}$-regular measures of $M(\underline{L})$.

$M_{\sigma}(\underline{L})=$ the set of $\sigma$-smooth measures on $\underline{L}$ of $M(\underline{L})$.

$M^{\sigma}(\underline{L})=$ the set of $\sigma$-smooth measures on $A(\underline{L})$ of $M(\underline{L})$.

$M_{R}{ }^{\sigma}(\underline{L})=$ the set of $\underline{L}$-regular measures of $M^{\sigma}(\underline{L})$.

Note that if $u \in M_{R}(\underline{L})$ and $u \in M_{\sigma}(\underline{L})$ then $u \in M_{R}{ }^{\sigma}(\underline{L})$.

Also we denote by $I(\underline{L}), I_{R}(\underline{L}), I_{\sigma}(\underline{L}), I^{\sigma}(\underline{L})$, and $I_{R}^{\sigma}(\underline{L})$ the subsets of $M(\underline{L}), M_{R}(\underline{L}), M_{\sigma}(\underline{L})$, $M^{\sigma}(\underline{L})$, and $M_{R}{ }^{\sigma}(\underline{L})$ consisting of zero-one valued measures.

Now for $u_{1}, u_{2} \in I(\underline{L}), u_{1} \leq u_{2}(\underline{L})$ means $u_{1}(L) \leq u_{2}(L)$ for $L \in \underline{L}$.

Let $J(\underline{L})$ denote those $u \in I(\underline{L})$ such that whenever $L_{n} \in \underline{L}, n=1,2, \ldots$ and $\bigcap_{n=1}^{\infty} L_{n} \in \underline{L}$ then $u\left(\bigcap_{n=1}^{\infty} L_{n}\right)=\inf _{n} u\left(L_{n}\right)$.

Clearly, $I^{\sigma}(\underline{L}) \subset J(\underline{L}) \subset I_{\sigma}(\underline{L})$.

For $u \in M(\underline{L})$ the support of $u, S(u)=\cap\{L \in \underline{L} \mid u(L)=u(X)\} . \underline{L}$ is replete if for any $u \in I_{R}^{\sigma}(\underline{L}), u \neq 0$, $S(u) \neq \phi$.

Let $C(\underline{L})$ be the set of all real-valued $\underline{L}$-continuous functions defined on $X$, where $f: X \rightarrow R$ is called $\underline{L}$-continuous if $f^{-1}(E) \in \underline{L}$ for any closed set $E \subset R$. If $X$ is a topological space, $C(X)$ denotes the continuous functions on $X$ or equivalently we can write $C(X)=C(F)$ where $F$ is the lattice of closed sets of $X, z(\underline{L})$ is the lattice of zero sets of functions in $C(\underline{L})$.

$C_{b}(\underline{L})=$ set of all real valued bounded $\underline{L}$-continuous functions defined on $X$.

Next we define $w(A)=\left\{u \in I_{R}(\underline{L}) \mid u(A)=1\right\}$ for $A \in A(\underline{L})$, and $w(\underline{L})=\{w(L) \mid L \in \underline{L}\}$.

We have for $A, B \in A(\underline{L})$ :

(1) $\quad w(A \cup B)=w(A) \cup w(B)$

(2) $\quad w(A \cap B)=w(A) \cap w(B)$

(3) $\quad w(A)^{\prime}=w\left(A^{\prime}\right)$

(4) $\quad w(A(\underline{L}))=A(w(\underline{L}))$

(5) $A \subset B=>w(A) \subset w(B)$

Note $w(\underline{L})$ is a lattice and if $\underline{L}$ is disjunctive then $w(A)=w(B)$ if and only if $A=B$.

The Wallman topology is obtained by taking $w(\underline{L})$ as a base for the closed sets of a topology on $I_{R}(\underline{L}) . \quad\left\langle I_{R}(\underline{L}), \tau w(\underline{L})\right\rangle$ is the general Wallman space associated with $X$ and $\underline{L}$. Note we have $w(L)=\bar{L}$ for $L \in \underline{L}$ if $\underline{L}$ is separating and disjunctive. We also define: $w_{\sigma}(A)=\left\{u \in I_{R}^{\sigma}(\underline{L}) \mid u(A)=1\right\}$ where $A \in A(\underline{L})$, and note $w(\underline{L}) \cap I_{R}{ }^{\sigma}(\underline{L})=w_{\sigma}(\underline{L})$.

We now consider two lattices. Let $\underline{L}_{1}$ and $\underline{L}_{2}$ denote lattices of subsets. of $X$ where $\underline{L}_{1} \subset \underline{L}_{2}$. $\underline{L}_{1}$ semi-separates $\underline{L}_{2}$ if $A \in \underline{L}_{1}, B \in \underline{L}_{2}$ and $A \cap B=\phi$ implies there exists $C \in \underline{L}_{1}, B \subset C$ and $A \cap C=\phi . \quad \underline{L}_{1}$ separates $\underline{L}_{2}$ if $A, B \in \underline{L}_{2}$ and $A \cap B=\phi$ implies there exists $C, D \in \underline{L}_{1}$ such that $A \subset C, B \subset D$, and $C \cap D=\phi . \quad \underline{L}_{2}$ is $\underline{L}_{1}$-countable paracompact if for every sequences $\left\{B_{n}\right\}$ of sets of $\underline{L}_{2}$, such that $B_{n} \downarrow \phi$ there exists $\left\{A_{n} \in \underline{L}_{1}\right\}$ such that $A_{n}{ }^{\prime} \downarrow \phi$ and $B_{n} \subset A_{n}{ }^{\prime}$. 
$\underline{L}_{2}$ is $\underline{L}_{1^{-}} c b$ if given $B_{n} \downarrow \phi, B_{n} \in \underline{L}_{2}$ there exists $\left\{A_{n}\right\}, A_{n} \in \underline{L}_{1}$ such that $A_{n} \downarrow \phi$ and $B_{n} \subset A_{n}$. Clearly if $\underline{L}_{1}$ separates $\underline{L}_{2}$ then $\underline{L}_{1}$ semiseparates $\underline{L}_{2}$.

If $\nu \in M\left(\underline{L}_{2}\right)$ then by $\nu \mid A\left(\underline{L}_{1}\right)$ we mean $\nu$ restricted to $A\left(\underline{L}_{1}\right)$. We state the following well known results:

Let $\underline{L}_{1} \subset \underline{L}_{2}$ be two lattices of subsets of $X$. If $\underline{L}_{1}$ semiseparates $\underline{L}_{2}$ then for $\nu \in M_{R}\left(\underline{L}_{2}\right)$, $u=\nu \mid A\left(\underline{L}_{1}\right) \in M_{R}\left(\underline{L}_{1}\right)$.

Suppose $\underline{L}_{1} \subset \underline{L}_{2}$ are two lattices of subsets of $X$. Then if $u \in M_{R}\left(\underline{L}_{1}\right), u$ extends to $\nu \in M_{R}\left(\underline{L}_{2}\right)$. Moreover, the extension is unique if $\underline{L}_{1}$ separates $\underline{L}_{2}$.

We will frequently assume in the sequel that $\underline{L}_{1} \subset \underline{L}_{2}$ and $\underline{L}_{2}$ is $\underline{L}_{1}$ countably paracompact or countably bounded, but we note that this is unnecessary in certain situations as the following facts listed below show:

(1) If $\underline{L}_{2}$ is $\underline{L}_{1}$ countably bounded and if $\underline{L}_{1}$ is countably paracompact (e.g., if $\underline{L}_{1}$ is complement generated) then $\underline{L}_{2}$ is $\underline{L}_{1}$ countably paracompact.

(2) If $\underline{L}_{2}$ is countably paracompact and if $\underline{L}_{1}$ separates $\underline{L}_{2}$ then $\underline{L}_{2}$ is $\underline{L}_{1}$ countably paracompact.

(3) Suppose $\underline{L}_{2}$ is $\underline{L}_{1}$ countably paracompact and $\underline{L}_{1}$ semiseparates $\underline{L}_{2}$ then $\underline{L}_{2}$ is $\underline{L}_{1}$ countably bounded.

(4) If $\underline{L}_{2}$ is countably paracompact and if $\underline{L}_{1}$ separates $\underline{L}_{2}$ then $\underline{L}_{2}$ is $\underline{L}_{1}$ countably bounded. 2.(b)NORMAL LATTICES AND MEASURES.

In this section we will consider a number of measure implications of normal lattices and other special lattices as well as converse implications. We first note:

THEOREM 2.1. Let $\underline{L}$ be a complemented generated lattice. The $u \in I_{\sigma}\left(\underline{L}^{\prime}\right)$ implies $u \in I_{R}{ }^{\sigma}(\underline{L})$.

PROOF. Since $\underline{L}$ is complemented generated then $\underline{L}$ is countably paracompact and therefore $I_{\sigma}\left(\underline{L}^{\prime}\right) \subset I_{\sigma}(\underline{L})$. Therefore it suffices to show $u \in I_{R}(\underline{L})$, but this is easy for if $L \in \underline{L}$ then $L=\bigcap_{n=1}^{\infty} L_{n}{ }^{\prime}, L_{n} \in \underline{L}$ all $n$, and we may assume that the $L_{n}{ }^{\prime} \downarrow \phi$. Now if $u(L)=0$, and if all $u\left(L_{n}{ }^{\prime}\right)=1$

then $\bigcap_{n=1}^{\infty} L_{n}{ }^{\prime} \cap L^{\prime}=\phi$ and $u\left(L_{n}{ }^{\prime} \cap L^{\prime}\right)=1$ all $n$ which is a contradiction since $u \in I_{\sigma}\left(\underline{L}^{\prime}\right)$. It follows that $u(L)=\inf \left\{u\left(\tilde{L}^{\prime}\right) \mid L \subset \tilde{L}^{\prime}, \tilde{L} \in \underline{L}\right\}$ and this implies $u \in I_{R}(\underline{L})$.

REMARK. It is equally easy to show if $\underline{L}$ is complement geuerated and $u \in M_{\sigma}\left(\underline{L}^{\prime}\right)$ then $u \in M_{R}{ }^{\sigma}(\underline{L})$.

THEOREM 2.2. Let $u \in J(\underline{L})$ and let $\underline{L}$ be a $\delta$-lattice then $u\left({\underset{i}{i=1}}_{i}^{\infty} L_{i}^{\prime}\right) \leq \sum_{i=1}^{\infty} u\left(L_{i}{ }^{\prime}\right)$ where all $L_{i} \in \underline{L}$.

PROOF. Suppose $u\left(\bigcup_{i=1}^{\infty} L_{i}{ }^{\prime}\right)=1$ and $\sum_{i=1}^{\infty} u\left(L_{i}{ }^{\prime}\right)=0$. Now $\sum_{i=1}^{\infty} u\left(L_{i}{ }^{\prime}\right)=0$ implies $u\left(L_{i}{ }^{\prime}\right)=0$ all $i$ and $\bigcap_{i=1}^{\infty} L_{i}=\left(\bigcup_{i=1}^{\infty} L_{i}^{\prime}\right)^{\prime}$ therefore $u\left(\bigcap_{i=1}^{\infty} L_{i}\right)=0$ where obviously $\prod_{i=1}^{\infty} L_{i} \in \underline{L}$. Also $u\left({ }_{i=1}^{\infty} L_{i}\right)=\inf u\left(L_{i}\right)$ since $u \in J(\underline{L})$. So $u\left(\bigcap_{i=1}^{\infty} L_{i}\right)=0$ implies there exists an $i_{0}$ such that $\bigcap_{i=1}^{\infty} L_{i} \subset L_{i_{0}}$ and $u\left(L_{i_{0}}\right)=0$. Therefore $u\left(L_{i_{0}}{ }^{\prime}\right)=1$ which is a contradiction, therefore theorem is proved.

THEOREM 2.3. If $\underline{L}$ is normal and complement generated then $u \in J(\underline{L})=>u \in I_{R}{ }^{\sigma}(\underline{L})$.

PROOF. Let $u \in J(\underline{L})$; we know that $u \leq \nu$ on $\underline{L}$ where $\nu \in I_{R}(\underline{L})$. This gives $\nu \leq u$ on $\underline{L}^{\prime}$. Suppose $u \neq \nu$. Then there exists $L \in \underline{L}$ such that $u(L)=0, \nu(L)=1$. However, $L=\bigcap_{n=1}^{\infty} L_{n}{ }^{\prime}$ since $\underline{L}$ is complement generated so $L \subset L_{n}{ }^{\prime}$. Therefore $\nu(L)=1=>\nu\left(L_{n}{ }^{\prime}\right)=1$ for all $n$ which implies $u\left(L_{n}{ }^{\prime}\right)=1$ for all $n$ as $\nu \leq u$ on $\underline{L}^{\prime}$. Now $L=\cap L_{n}{ }^{\prime}=>L \cap L_{n}=\phi$ therefore since $\underline{L}$ is normal there exists $A_{n}{ }^{\prime}, B_{n}{ }^{\prime} \in \underline{L}^{\prime}$ such that $L \subset A_{n}{ }^{\prime}, L_{n} \subset B_{n}{ }^{\prime}$, and $A_{n}{ }^{\prime} \cap B_{n}{ }^{\prime}=\phi$. Therefore $L \subset A_{n}{ }^{\prime} \subset B_{n} \subset L_{n}$ from this 
which gives $\nu\left(A_{n}{ }^{\prime}\right)=1$ and $\nu\left(B_{n}\right)=1$ by monotonicity of $\nu$. Therefore $u\left(B_{n}\right)=1$ as $u \leq \nu$ on $\underline{L}$. Also $L \subset A_{n}{ }^{\prime} \subset B_{n} \subset L_{n}{ }^{\prime}=>L \subset \bigcap_{n=1}^{\infty} A_{n}{ }^{\prime} \subset \bigcap_{n=1}^{\infty} B_{n} \subset \bigcap_{n=1}^{\infty} L_{n}{ }^{\prime}=L$ which implies that $L=\bigcap_{n=1}^{\infty} A_{n}{ }^{\prime}=\bigcap_{n=1}^{\infty} B_{n}=$ $\bigcap_{n=1}^{\infty} L_{n}^{\prime}$, so $u(L)=0=>u\left(\bigcap_{n=1}^{\infty} B_{n}\right)=0$ which $=>u\left(B_{n}\right)=0$ by $u \in J(\underline{L})$. This is a contradiction as $u\left(B_{n}\right)=1$. Therefore $u=v \in I_{R}(\underline{L})=>u \in I_{R}(\underline{L})$. Now $u \in J(\underline{L})=>u \in I^{\sigma}(\underline{L})$, therefore $u \in I_{R}^{\sigma}(\underline{L})$.

THEOREM 2.4. Let $\underline{L}$ be a normal lattice, $u \in I_{R}(\underline{L}), u \leq \rho\left(\underline{L}^{\prime}\right)$ where $\rho \in I_{R}\left(\underline{L}^{\prime}\right)$. Then for $L \in \underline{L}, u\left(L^{\prime}\right)=\sup \left\{\rho(\tilde{L})\left|\tilde{L} \subset L^{\prime}\right| \tilde{L} \in \underline{L}\right\}$.

PROOF. Suppose $u\left(L^{\prime}\right)=1$, where $L \in \underline{L}$ then since $u \in I_{R}(\underline{L})$ there exists $\tilde{L} \subset L^{\prime}, \tilde{L} \in \underline{L}, u(\tilde{L})=1$. Since $\tilde{L} \subset L^{\prime}=>L \cap \tilde{L}=\phi$, therefore by normality there exists $A, B \in \underline{L}$ such that $L \subset A^{\prime}, \tilde{L} \subset B^{\prime}, A^{\prime} \cap B^{\prime}=\phi$. Therefore $\tilde{L} \subset B^{\prime} \subset A \subset L^{\prime}$, also $u(\tilde{L})=1=>u\left(B^{\prime}\right)=1$ by monotonicity of $u$. Therefore $\rho\left(B^{\prime}\right)=1$ as $u \leq \rho\left(\underline{L}^{\prime}\right) . \quad \rho(A)=1$ follows by monotonicity of $\rho$, proving the theorem.

REMARK. This theorem is equivalent to the following: Let $\underline{L}$ be normal and let $\nu \leq u(\underline{L})$ where $\nu \in I(\underline{L})$ and $u \in I_{R}(\underline{L})$. Then $u\left(L^{\prime}\right)=\sup \left\{\nu(\tilde{L}) \mid \tilde{L} \subset L^{\prime}, \tilde{L} \in \underline{L}\right\}$. Next we show that actually the property in Theorem 2.4 or equivalently the one in the remark characterizes normal lattices, i.e.,

THEOREM 2.5. Suppose $u \in I_{R}(\underline{L})$ and $\rho \leq u(\underline{L})$ where $\rho \in I_{R}\left(\underline{L}^{\prime}\right)$ and $u\left(L^{\prime}\right)=1, L \in \underline{L}$ implies $L^{\prime} \supset A \in \underline{L}$ such that $\rho(A)=1$. Then $\underline{L}$ is normal.

PROOF. Let $\rho \leq u(\underline{L}), \rho \leq \nu(\underline{L})$ where $u, \nu \in I_{R}(\underline{L})$ and $\rho \in I_{R}\left(\underline{L}^{\prime}\right)$. Assume $u \neq \nu$, this implies $u\left(L_{1}\right)=0, v\left(L_{1}\right)=1, u\left(L_{2}\right)=1, v\left(L_{2}\right)=0$ where $L_{1}, L_{2} \in \underline{L}$ and $L_{1} \cap L_{2}=\phi$. Now $u\left(L_{1}\right)=0$ implies $u\left(\dot{L}_{1}^{\prime}\right)=1$ which implies there exists $L_{1}^{\prime} \supset A \in \underline{L}$ such that $\rho(A)=1$ and $v\left(L_{2}\right)=0$ implies $v\left(L_{2}^{\prime}\right)=1$ which implies there exists $L_{2}^{\prime} \supset B \in \underline{L}$ such that $\rho(B)=1$. Since $A \subset L_{1}^{\prime}, B \subset L_{2}^{\prime}$ then $L_{1} \subset A^{\prime}$ and $L_{2} \subset B^{\prime}$. So $\rho(B)=1$ implies $\rho\left(B^{\prime}\right)=0$ which implies $u\left(B^{\prime}\right)=0$ as $u \leq \rho\left(\underline{L}^{\prime}\right)$. However, by monotonicity $u\left(L_{2}\right) \leq u\left(B^{\prime}\right)$ and $u\left(L_{2}\right)=1$ which implies $u\left(B^{\prime}\right)=1$ which contradicts $u\left(B^{\prime}\right)=0$. Therefore $u=v$ which means $\underline{L}$ is normal.

THEOREM 2.6. Let $\underline{L}$ be a normal lattice, $u \in I_{\sigma}(\underline{L}), u \leq v(\underline{L})$ where $\nu \in I_{R}(\underline{L})$. Then $\nu \in I_{\sigma}\left(\underline{L}^{\prime}\right)$.

PROOF. Suppose $u \in I_{\sigma}(\underline{L})$ we know $\rho \leq u \leq \nu(\underline{L})$ where $v \leq u \leq \rho\left(\underline{L}^{\prime}\right)$ and $\nu \in I_{R}(\underline{L}), \rho \in I_{R}(\underline{L})$. Suppose $L_{n}^{\prime} \downarrow \phi, v\left(L_{n}^{\prime}\right)=1$ all $n, L_{n}^{\prime} \in \underline{L}^{\prime}$. Then there exists $\tilde{L}_{n} \subset L_{n}^{\prime}$ such that $\rho\left(\tilde{L}_{n}\right)=1$ all $n$ by Theorem 2.5. Therefore $u\left(\tilde{L}_{n}\right)=1$ since $\rho \leq u(\underline{L})$. Now $\tilde{L}_{n} \downarrow \phi$ since $\bigcap_{n=1}^{\infty} \tilde{L}_{n} \subset \bigcap_{n=1}^{\infty} L_{n}^{\prime}$. This contradicts the fact that $u \in I_{\sigma}(\underline{L})$, therefore $v \in I_{\sigma}\left(\underline{L}^{\prime}\right)$.

COROLLARY 2.7. If $\underline{L}$ is normal and countably paracompact then the $\nu$ (from Theorem 2.6) belongs to $I_{R}{ }^{\sigma}(\underline{L})$.

PROOF. Since $\underline{L}$ is countably paracompact then $I_{\sigma}\left(\underline{L}^{\prime}\right) \subset I_{\sigma}(\underline{\dot{L}})$ by Theorem 2.2. Then $\nu \in I_{\sigma}(\underline{L})$ and since $\nu \in I_{R}(\underline{L})$ it follows that $\nu \in I_{R}{ }^{\sigma}(\underline{L})$.

Next we consider a pair of lattices $\underline{L}_{1}, \underline{L}_{2}$ of $X$ such that $\underline{L}_{1} \subset \underline{L}_{2}$, then we have:

THEOREM 2.8. If $\underline{L}_{1}$ separates $\underline{L}_{2}$ then $\underline{L}_{1}$ is normal if and only if $\underline{L}_{2}$ is normal.

PROOF. The proof is not difficult. We just show $\underline{L}_{2}$ normal implies $\underline{L}_{1}$ normal. Now let $\underline{L}_{2}$ be normal and $u \in I\left(\underline{L}_{1}\right), u \leq v_{1}\left(\underline{L}_{1}\right), u \leq v_{2}\left(\underline{L}_{1}\right)$ where $v_{1}, v_{2} \in I_{R}\left(\underline{L}_{1}\right)$. Now we can extend $u \in I\left(\underline{L}_{1}\right)$ to $\lambda \in I\left(\underline{L}_{2}\right)$ and extend $v_{1}$ to $\tau_{1} \in I_{R}\left(\underline{L}_{2}\right), v_{2}$ to $\tau_{2} \in I_{R}\left(\underline{L}_{2}\right)$. Now we have $\lambda \leq \tau_{1}\left(\underline{L}_{2}\right), \lambda \leq \tau_{2}\left(\underline{L}_{2}\right)$ which is not difficult to see since $\underline{L}_{1}$ separates $\underline{L}_{2}$. Now $\underline{L}_{2}$ is normal, therefore $\tau_{1}=\tau_{2}$ and $\nu_{1}=\tau_{1}\left|A\left(\underline{L}_{1}\right)=\tau_{2}\right| A\left(\underline{L}_{1}\right)=\nu_{2}$. Therefore $\underline{L}_{1}$ is normal.

\section{THE WALLMAN SPACE $I_{R}(\underline{L})$.}

We give here a brief discussion of the general Wallman space (see also [11]). Consider the set $I_{R}(\underline{L})$ and the lattice of subsets $w(\underline{L})$. It is well-known that $w(\underline{L})$ is compact and it is not difficult to show: 
THEOREM 3.1. The following are equivalent:

(a) $w(\underline{L})$ is normal;

(b) $w(\underline{L})$ is regular;

(c) $w(\underline{L})$ is $T_{2}$.

Now since $w(\underline{L})$ is compact, $\tau w(\underline{L})$ the topology of closed sets, is compact and $w(\underline{L})$ separates $\tau w(\underline{L})$, and by Theorem $2.8 w(\underline{L})$ is normal if and only if $\tau w(\underline{L})$ is normal. $\left\langle I_{R}(\underline{L}), \tau w(\underline{L})\right\rangle$ is a compact topological space and it is always $T_{1}$. Assuming $\underline{L}$ is disjunctive, it is $T_{2}$ if and only if $\underline{L}$ is normal. Next, let $\underline{L}$ be a $\delta$-normal lattice of subsets of $X$, then the Alexandroff representation theorem (see [1]) yields for the conjugate space of $C_{b}(\underline{L})$, namely $C_{b}(\underline{L})^{\prime}=M_{R}(\underline{L})$ where to any $\Phi \in C_{b}(\underline{L})^{\prime}$ there corresponds a unique $u \in M_{R}(\underline{L})$ such that $\Phi(f)=\int_{X} f d u$, for all $f \in C_{b}(\underline{L})$.

A net $\left\{u_{\alpha}\right\}$ in $M_{R}(\underline{L})$ converges to $u$ in $M_{R}(\underline{L})$ in the weak * topology if and only if $\int_{X} f d u_{\alpha} \rightarrow \int_{X} f d u$ for all $f \in C_{b}(\underline{L})$. We shall denote weak * convergence by $\underline{w}_{\rightarrow}^{*}$.

THEOREM 3.2. Now let $\underline{L}$ be $\delta$-normal and consider convergence in $M_{R}{ }^{+}(\underline{L})$. The following are equivalent:

(1) $u_{\alpha} \stackrel{w^{*}}{\rightarrow} u$

(2) $u_{\alpha}(X) \rightarrow u(X)$ and $\overline{\lim _{\alpha}} u_{\alpha}(A) \leq u(A)$ for all $A \in \underline{L}$

(3) $u_{\alpha}(X) \rightarrow u(X)$ and $\frac{\lim }{\alpha} u_{\alpha}\left(A^{\prime}\right) \geq u\left(A^{\prime}\right)$ for all $A^{\prime} \in \underline{L}^{\prime}$. For the proof in this particular setting see $([7])$.

THEOREM 3.3. Let $u_{\alpha} \in I_{R}(\underline{L}) \stackrel{w^{*}}{\rightarrow} u \in M_{R}(\underline{L})$ then $u \in I_{R}(\underline{L})$. Thus $I_{R}(\underline{L})$ is $w^{*}$-closed in $M_{R}(\underline{L})$. PROOF. Suppose $u_{\alpha} \in I_{R}(\underline{L})^{w^{*}} \rightarrow u \in M_{R}(\underline{L})$. Therefore $u_{\alpha}(X) \rightarrow u(X)$ by Theorem 3.2. Now $u_{\alpha}(X)=1$ since $u_{\alpha} \in I_{R}(\underline{L})$, therefore $u(X)=1$, which means for $A \in A(\underline{L}): 0 \leq u(A) \leq 1$. Suppose $A \in A(\underline{L})$ and $0<u(A)<1$. Since $u \in M_{R}(\underline{L})$ there exists $L \in \underline{L} \subset A$ such that $0<u(L) \leq u(A)$ and there exists $A \subset \tilde{L}^{\prime} \in \underline{L}^{\prime}$ such that $u(A) \leq u\left(\tilde{L}^{\prime}\right)<1$. Therefore $0<u(L) \leq u\left(\tilde{L}^{\prime}\right)<1$. Now $L \subset \tilde{L}^{\prime}$ therefore $L \cap \tilde{L}=\phi$ which implies there exists $A, B \in \underline{L}$ such that $L \subset A^{\prime}, \tilde{L} \subset B^{\prime}, A^{\prime} \cap B^{\prime}=\phi$ by $\underline{L}$ normal. Therefore $L \subset A^{\prime} \subset B \subset \tilde{L}^{\prime}$ which implies $0<u(L) \leq u\left(A^{\prime}\right) \leq u(B) \leq u\left(\tilde{L}^{\prime}\right)<1$ so that $u\left(A^{\prime}\right) \leq u(B)<1$. Now since $u_{\alpha} \stackrel{w^{*}}{\rightarrow} u$ then $\varlimsup_{\alpha} u_{\alpha}(B) \leq u(B)$ for $B \in \underline{L}$. Now $u(B)<1$, therefore $\overline{\lim _{\alpha}} u_{\alpha}(B)<1$ which means $u_{\alpha}(B)=0$ since $u_{\alpha} \in I_{R}(\underline{L})$. Also $\frac{\lim }{\alpha} u_{\alpha}\left(A^{\prime}\right) \geq u\left(A^{\prime}\right)$ for $A^{\prime} \in \underline{L}^{\prime}$ but $u\left(A^{\prime}\right)<1$, therefore $\frac{\lim }{\alpha} u_{\alpha}\left(A^{\prime}\right)>0$ as $0<u\left(A^{\prime}\right)<1$. Therefore $u_{\alpha}\left(A^{\prime}\right)=1$ since $u_{\alpha} \in I_{R}(\underline{L})$. However for $A^{\prime} \subset B$ we have $u_{\alpha}\left(A^{\prime}\right)=1, u_{\alpha}(B)=0$ which is impossible. Therefore $u(A)=0$ or 1 , which implies $u \in I_{R}(\underline{L})$.

THEOREM 3.4. $\overline{\left[\left\{u_{x}\right\}\right]}=M_{R}(\underline{L})$

PROOF. The proof of this is not difficult and can be modelled after the well-known special case of $\underline{L}$ being the lattice of zero sets in a Tychonoff space.

THEOREM 3.5. The $w^{*}$-topology of $M_{R}(\underline{L})$ when restricted to $I_{R}(\underline{L})$ gives the Wallman topology $\tau w(\underline{L})$ for closed sets.

PROOF. Let $u_{\alpha} \stackrel{w^{*}}{\rightarrow} u$ we will show $u_{\alpha} \stackrel{w}{\rightarrow} u$ where $w$ is convergence in Wallman. Consider $u_{0} \in w(L)^{\prime}$, therefore $u_{0}\left(L^{\prime}\right)=1$. Using Theorem 3.2 we have $\frac{\lim }{\alpha} u_{\alpha}\left(L^{\prime}\right) \geq u_{0}\left(L^{\prime}\right)$, therefore $\frac{\lim }{\alpha} u_{\alpha}\left(L^{\prime}\right)=1$. But $1=\frac{\lim }{\alpha} u_{\alpha}\left(L^{\prime}\right) \leq \lim _{\alpha} u_{\alpha}\left(L^{\prime}\right) \leq 1$, therefore $\lim _{\alpha} u_{\alpha}\left(L^{\prime}\right)=1$. So there exists $\alpha_{0}$ such that for all $\alpha \geq \alpha_{0}$ $u_{\alpha}\left(L^{\prime}\right)=1$, therefore $u_{\alpha} \in w\left(L^{\prime}\right)$ for all $\alpha \geq \alpha_{0}$. This gives $u_{\alpha} \underset{w}{\rightarrow} u$ which proves the theorem.

We assume now that $\underline{L}$ is $\delta$-normal, separating and disjunctive. Let $f \in C_{b}(\underline{L})$ we define $\hat{f}$ on $I_{R}(\underline{L})$ by $\hat{f}(u)=\int_{X} f d u$ where $u \in I_{R}(\underline{L})$.

THEOREM 3.6. $\hat{f} \in C\left(I_{R}(\underline{L})\right)$. 
PROOF. Let $u_{\alpha} \stackrel{w}{\rightarrow} u_{0}$. We must show that $\hat{f}\left(u_{\alpha}\right) \rightarrow \widehat{f}\left(u_{0}\right)$ which means $u_{\alpha} \stackrel{w^{*}}{\rightarrow} u_{0}$. For $u_{0} \in w\left(L^{\prime}\right)$ we have $u_{\alpha} \in w\left(L^{\prime}\right)$ for all $\alpha \geq \alpha_{0}$ as $u_{\alpha} \stackrel{w}{\rightarrow} u_{0}$. Therefore, $u_{\alpha}\left(L^{\prime}\right)=1, \alpha \geq \alpha_{0}$, which implies $\lim _{\alpha} u_{\alpha}\left(L^{\prime}\right)=1$. Therefore $\frac{\lim }{\alpha} u_{\alpha}\left(L^{\prime}\right)=\prod_{\alpha} u_{\alpha}\left(L^{\prime}\right)=\lim u_{\alpha}\left(L^{\prime}\right)=1$ and $u_{0}\left(L^{\prime}\right)=1$ as $u_{0} \in w\left(L^{\prime}\right)$. So $\frac{\lim }{\alpha} u_{\alpha}\left(L^{\prime}\right) \geq u_{0}\left(L^{\prime}\right)$ and therefore by Theorem 3.2 we have $u_{\alpha} \stackrel{w^{*}}{\rightarrow} u_{0}$ which proves $\hat{f} \in C\left(I_{R}(\underline{L})\right)$.

THEOREM 3.7. The correspondence $f \rightarrow \hat{f}$ is a bijection between $C_{b}(\underline{L})$ and $C\left(I_{R}(\underline{L})\right)$; the continuous functions on the Wallman space $I_{R}(\underline{L})$.

PROOF. Let $A=\left\{\hat{f} \mid f \in C_{b}(\underline{L})\right\}$. Then $A \subset C(\tau w(\underline{L}))=C\left(I_{R}(\underline{L})\right)$. Since $u_{\alpha} \stackrel{w^{*}}{\rightarrow} u \Rightarrow \widehat{f}(u) \in C\left(I_{R}(\underline{L})\right)$. Now it is easy to show the following:

(1) $\hat{f+g}=\widehat{f}+\widehat{g}$

(2) $\hat{a} \hat{f}=a \hat{f}$ for $a \in R$

(3) $\hat{f} \dot{g}=\hat{f} \hat{g}$

(4) $\|\hat{f}\|=\|f\|$, therefore $A$ is a closed subalgebra of $C(\tau w(\underline{L}))$

(5) $A$ separates points. We can prove this by showing for $u, v \in I_{R}(\underline{L}), u \neq v$ there exists $\hat{f} \in A$ such that $\hat{f}(u)=1$ and $\hat{f}(v)=0$. This is done by using the normality of $\underline{L}$.

(6) $1 \in A$. Therefore given $u \in I_{R}(\underline{L})$ there exists $\widehat{f} \in A$ such that $\hat{f}(u) \neq 0$.

So by the Stone-Weierstrass theorem $A=\bar{A}=C(\tau w(\underline{L}))$ which proves the theorem.

4. THE SPACE $Q(\underline{L})$.

In this section, we consider the important measures of $I_{R}(\underline{L})$ which integrate all $f \in C(\underline{L})$ and consider their relationship to $I_{R}{ }^{\sigma}(\underline{L})$. Let $\underline{L}$ be $\delta$-normal lattice. We define $Q(\underline{L})=\left\{u \in I_{R}(\underline{L})\left|\int_{X}\right| f \mid d u<\infty\right.$ for all $\left.f \in C(\underline{L})\right\}$.

THEOREM 4.1. $I_{R}{ }^{\sigma}(\underline{L}) \subset Q(\underline{L})$.

PROOF. Let $v \in I_{R}{ }^{\sigma}(\underline{L})$ and $L_{n}=(|f| \geq n)$. One can see $L_{n} \downarrow \phi$ which implies $v\left(L_{n}\right) \rightarrow 0$ since $v \in I_{R}{ }^{\sigma}(\underline{L})$. Therefore $v\left(L_{N}\right)=0$ for $N$ big. Now

$$
\begin{gathered}
\int_{X}|f| d v=\int_{L_{N}}|f| d v+\int_{L_{N^{\prime}}}|f| d v \\
\leq N V L_{N^{\prime}} \\
\leq N
\end{gathered}
$$

Therefore $\int_{X}|f| d v \leq N$ which proves $v \in Q(\underline{L})$.

THEOREM 4.2. $I_{R}(\underline{L}) \cap I_{\sigma}\left(\underline{L}^{\prime}\right) \subset Q(\underline{L})$.

PROOF. Let $(|f|>n)=A_{n}^{\prime}$. Clearly $A_{n}^{\prime} \downarrow \phi$ and $A_{n} \in \underline{L}$ for all $n$. Now let $v \in I_{R}(\underline{L}) \cap I_{\sigma}\left(\underline{L}^{\prime}\right)$, therefore $v\left(A_{n}^{\prime}\right)=0, n \geq N$. Now $\int_{X}|f| d v=\int_{A_{N}}|f| d v+\int_{A_{N^{\prime}}}|f| d v$. Therefore $\int_{X}|f| d v \leq N V\left(A_{N}\right)$, so $\int_{X}|f| d v \leq N V\left(A_{N}\right)<\infty$. Therefore, $v \in Q(\underline{L})$ which provides the theorem.

THEOREM 4.3. $I_{R}{ }^{\sigma}(\underline{L}) \subset I_{R}(\underline{L}) \cap I_{\sigma}\left(\underline{L}^{\prime}\right) \subset Q(\underline{L})$.

PROOF. By Theorems 4.1 and 4.2 and the trivial observation that $I_{R}^{\sigma}(\underline{L}) \subset I_{R}(\underline{L}) \cap I_{\sigma}\left(\underline{L}^{\prime}\right)$, the result is proved.

Following Varadarajan who considered the lattice of zero sets in a Tychonoff space, we introduce

DEFINITION. The Sequence $\left\{B_{n}\right\}$ in $\underline{L}$ is called regular if $B_{n}{ }^{\prime} \downarrow \phi$ and there exists $A_{n}$ in $\underline{L}$ such that $B_{n} \subset A_{n}{ }^{\prime} \subset B_{n+1}$ for all $n$.

THEOREM 4.4. Let $\left\{B_{n}\right\}$ be a regular sequence. Then there exists $\left\{f_{n}\right\}, f_{n} \in C_{b}(\underline{L}), 0 \leq f_{n} \leq 1$ such that $f_{n} \downarrow \phi, f_{n}\left(B_{n}\right)=0, f_{n}\left(B_{n+1}^{\prime}\right)=1$ for $n=1,2, \ldots$

PROOF. Omitted. 
THEOREM 4.5. Let $X$ be an abstract set and $\underline{L}$ a $\delta$-normal lattice of subsets which is also countably paracompact. Let $\left\{A_{n}\right\}$ in $\underline{L}, A_{n} \downarrow \phi$. Then there exists a regular sequence $\left\{C_{n}\right\}$ such that $C_{n} \subset A_{n}^{\prime}$ for all $n$.

PROOF. Since $A_{n} \downarrow \phi$ and since $\underline{L}$ is countably paracompact then there exists $\left\{B_{n}\right\}$ in $\underline{L}$ with $A_{n} \subset B_{n}^{\prime} \downarrow \phi$. Now we show by induction that for any $n$ we have $\left\{C_{K}\right\},\left\{D_{K}\right\}$ in $\underline{L}$ with $A_{K} \subset C_{K}{ }^{\prime} \subset D_{K} \subset\left(B_{K}{ }^{\prime} \cap C_{K-1}^{\prime}\right)$ where $K=1, \ldots n$ : (1) For $n=1$, take $C_{0}=\phi$, and $A_{1} \subset C_{1}{ }^{\prime} \subset D_{1} \subset B_{1}{ }^{\prime}$ follows by normality. (2) Assume expression is true for $n$. Now $A_{n+1} \subset B_{n+1}^{\prime}$ and $A_{n+1} \subset A_{n} \subset C_{n}^{\prime}$, therefore $A_{n+1} \subset B_{n+1}^{\prime} \cap C_{n}^{\prime}$. Using normality, there exists $C_{n+1}, D_{n+1} \in \underline{L}$ such that $A_{n+1} \subset C_{n+1}^{\prime} D_{n+1} \subset\left(B_{n+1}^{\prime} \cap C_{n}^{\prime}\right)$ which finishes the induction argument. Since $C_{n} \subset A_{n}^{\prime}$ we must show $\left\{C_{n}\right\}$ is regular. Now $C_{n}{ }^{\circ} \subset B_{n}^{\prime}$ implies $C_{n}{ }^{\prime} \downarrow \phi$ and $C_{n} \subset D_{n+1}^{\prime} \subset C_{n+1}$. Therefore $\left\{C_{n}\right\}$ is regular as $D_{n+1} \in \underline{L}$. Finally using the previous two results it is not difficult to show using an argument similar to Varadarajan that the following holds:

THEOREM 4.6. Let $\underline{L}$ be $\delta$-normal and countably paracompact, then $Q(\underline{L}) \subset I_{R}{ }^{\sigma}(\underline{L})$.

So using Theorems 4.1 and 4.6 we have:

THEOREM 4.7. Let $\underline{L}$ be $\delta$-normal and countably paracompact, then $Q(\underline{L})=I_{R}{ }^{\sigma}(\underline{L})$.

We also have:

THEOREM 4.8. If $Q(\underline{L})=I_{R}(\underline{L}) \cap I_{\sigma}\left(\underline{L}^{\prime}\right)$ and if $I_{\sigma}\left(\underline{L}^{\prime}\right) \subset I_{\sigma}(\underline{L})$ then $Q(\underline{L})=I_{R}{ }^{\sigma}(\underline{L})$.

PROOF. $Q(\underline{L})=I_{R}(\underline{L}) \cap I_{\sigma}\left(\underline{L}^{\prime}\right) \subset I_{R}(\underline{L}) \cap I_{\sigma}(\underline{L})$, but we know if $v \in M_{R}(\underline{L})$ and $v \in M_{\sigma}(\underline{L})$ then $v \in M_{R}{ }^{\sigma}(\underline{L})$. Therefore $Q(\underline{L}) \subset I_{R}(\underline{L}) \cap I_{\sigma}(\underline{L})=I_{R}{ }^{\sigma}(\underline{L})$, so $Q(\underline{L}) \subset I_{R}{ }^{\sigma}(\underline{L})$. However, from Theorem 4.1 we have $I_{R}{ }^{\sigma}(\underline{L}) \subset Q(\underline{L})$, therefore $Q(\underline{L})=I_{R}{ }^{\sigma}(\underline{L})$.

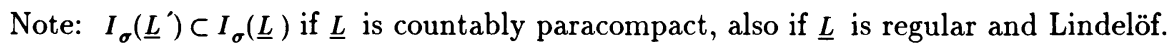

Now we consider two lattices $\underline{L}_{1}$ and $\underline{L}_{2}$ such that $\underline{L}_{1} \subset \underline{L}_{2}$. Then $C\left(\underline{L}_{1}\right) \subset C\left(\underline{L}_{2}\right)$.

THEOREM 4.9. Let $\underline{L}_{1}, \underline{L}_{2}$ be lattices of subsets such that $\underline{L}_{1}$ semi-separates $\underline{L}_{2}$. If $v \in Q\left(\underline{L}_{2}\right)$ and if $u=\nu \mid A\left(\underline{L}_{1}\right)$, then $u \in Q\left(\underline{L}_{1}\right)$.

PROOF. Since $\underline{L}_{1}$ semi-separates $\underline{L}_{2}, u \in I_{R}\left(\underline{L}_{1}\right)$. Also, since $C\left(\underline{L}_{1}\right) \subset C\left(\underline{L}_{2}\right)$ and since v integrates all $f \in C\left(\underline{L}_{2}\right), u$ integrates all $g \in C\left(\underline{L}_{1}\right)$. Hence $u \in Q\left(\underline{L}_{1}\right)$.

THEOREM 4.10. Let $\underline{L}_{1}, \underline{L}_{2}$ be lattice of subsets such that $\underline{L}_{1}$ separates $\underline{L}_{2}$. Let $v \in Q\left(\underline{L}_{2}\right)$ and $u=\nu \mid A\left(\underline{L}_{1}\right)$. If $Q\left(\underline{L}_{1}\right)=I_{R}{ }^{\sigma}\left(\underline{L}_{1}\right)$ then $v \in I_{\sigma}\left(\underline{L}_{2}{ }^{\prime}\right)$.

PROOF. By the previous theorem $u \in Q\left(\underline{L}_{1}\right)=I_{R}{ }^{\sigma}\left(\underline{L}_{1}\right)$ by hypothesis, and since $\underline{L}_{1}$ separates $\underline{L}_{2}$ it is easy to see $\nu$, the extension of $u$, is in $I_{\sigma}\left(\underline{L}_{2}^{\prime}\right)$.

THEOREM 4.11. Let $\underline{L}_{1}, \underline{L}_{2}$ be lattice of subsets such that ${ }^{\prime} \underline{L}_{1}$ separates $\underline{L}_{2}$. If $Q\left(\underline{L}_{1}\right)=I_{R}{ }^{\sigma}\left(\underline{L}_{1}\right)$ then $Q\left(\underline{L}_{2}\right)=I_{R}\left(\underline{L}_{2}\right) \cap I_{\sigma}\left(\underline{L}_{2}^{\prime}\right)$.

PROOF. $v \in Q\left(\underline{L}_{2}\right)$ implies $v \in I_{R}\left(\underline{L}_{2}\right)$, but $v \in I_{\sigma}\left(\underline{L}_{2}^{\prime}\right)$ from Theorem 4.10, therefore $Q\left(\underline{L}_{2}\right) \subset I_{R}\left(\underline{L}_{2}\right) \cap I_{\sigma}\left(\underline{L}_{2}^{\prime}\right)$. However we know if $v \in I_{R}\left(\underline{L}_{2}\right) \cap I_{\sigma}\left(\underline{L}_{2}^{\prime}\right)$ then $v \in Q\left(\underline{L}_{2}\right)$ from Theorem 4.2 which proves the result.

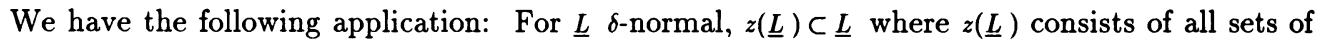
$\underline{L}$ of the form $L=\bigcap_{n=1}^{\infty} L_{n}^{\prime}, L_{n} \in \underline{L}$ for all $n$, (see [1]). Now $z(\underline{L})$ separates $\underline{L}$ and $z(\underline{L})$ is normal and countably paracompact. Therefore by Theorem 4.7 we have $I_{R}{ }^{\sigma}(z(\underline{L}))=Q(z(\underline{L}))$. Now using Theorem 4.11 we have $Q(\underline{L})=I_{R}(\underline{L}) \cap I_{\sigma}\left(\underline{L}^{\prime}\right)$. Also if $I_{\sigma}\left(\underline{L}^{\prime}\right) \subset I_{\sigma}(\underline{L})$ then $Q(\underline{L})=I_{R}{ }^{\sigma}(\underline{L})$ by Theorem 4.8.

REMARK. We recall that if $X$ is Tychonoff space and if $\underline{L}=z$, the lattice of zero sets then $\left(I_{R}{ }^{\sigma}(z), \tau w_{\sigma}(z)\right)$ is the realcompactification $\nu(X)$ of $X$.

Now we consider other criterion for $Q(\underline{L})=I_{R}(\underline{L}) \cap I_{\sigma}\left(\underline{L}^{\prime}\right)$. If $X$ is a topological space and if $A \subset X$ we denote by $\bar{A}^{\delta}$ the $G_{\delta}$-closure of $A$. Now if $X$ is an abstract set and $\underline{L}$ as usual is a 
separating disjunctive $\delta$-normal lattice of subsets then we can view $X$ embedded in $Q(\underline{L})$; we have $X \subset Q(\underline{L}) \subset I_{R}(\underline{L})$. In fact, using Theorem 4.3 we have $X \subset I_{R}{ }^{\sigma}(\underline{L}) \subset I_{R}(\underline{L}) \cap I_{\sigma}\left(\underline{L}^{\prime}\right) \subset Q(\underline{L}) \subset I_{R}(\underline{L})$.

THEOREM 4.12. $\bar{X}^{\delta} \subset Q(\underline{L})$ where $\bar{X}^{\delta}$ is the $G_{\delta}$-closure of $X$ in the Wallman space $I_{R}(\underline{L})$.

PROOF. Suppose $u \in \bar{X}^{\delta}$. If $u \notin Q(\underline{L})$ then there exists $f \in C(\underline{L}), f \geq 0$ such that $\int_{\infty} f d u=\infty$. Let $A_{n}^{\prime}=(f>n) \in \underline{L}^{\prime}$. Then $A_{n}^{\prime} \downarrow \phi$ and $u\left(A_{n}^{\prime}\right)=1$. Therefore $u \in \stackrel{\cap}{n}=1_{1}^{\infty} w\left(A_{n}{ }^{\prime}\right) \subset I_{R}(\underline{L})-X$ which contradicts the fact $u \in \bar{X}^{\delta}$. Therefore $u \in Q(\underline{L})$, so $\bar{X}^{\delta} \subset Q(\underline{L})$.

THEOREM 4.13. If $Q(\underline{L}) \subset \bar{X}^{\delta}$, then $G_{\delta}$-closure of $X$ in $I_{R}(\underline{L})$, then $u \in I_{\sigma}\left(\underline{L}^{\prime}\right)$ where $u \in Q(\underline{L})$.

PROOF. Suppose $u \in Q(\underline{L})$ which implies $u \in I_{R}(\underline{L})$. If $u \notin I_{\sigma}\left(\underline{L}^{\prime}\right)$ then there exists $L_{n}^{\prime} \downarrow \phi$, $L_{n} \in \underline{L}, u\left(L_{n}{ }^{\prime}\right)=1$. Therefore $u \in \stackrel{\cap}{n}=1_{n}^{\infty} w\left(L_{n}{ }^{\prime}\right) \subset I_{R}(\underline{L})-X$. Therefore $u \notin \bar{X}^{\delta}$, so $Q(\underline{L}) \subset \bar{X}^{\delta}$ implies $u \in I_{\boldsymbol{g}}\left(\underline{L}^{\prime}\right)$.

THEOREM 4.14. $Q(\underline{L})=\bar{X}^{\delta}$ if and only if $u \in I_{\sigma}\left(\underline{L}^{\prime}\right)$ for all $u \in Q(\underline{L})$.

PROOF. If $Q(\underline{L})=\bar{X}^{\delta}$ and if $u \in Q(\underline{L})$ then $u \in I_{\sigma}\left(\underline{L}^{\prime}\right)$ by the previous theorem. While if $Q(\underline{L}) \subset I_{\sigma}\left(\underline{L}^{\prime}\right)$ then we must have $Q(\underline{L}) \subset \bar{X}^{\delta}$ for if not then there exists $G \in G_{\delta}$ such that $u \in G \subset I_{R}(\underline{L})-X$ where $u \in I_{R}(\underline{L})$. Therefore $u \in \stackrel{\cap}{n}=1_{n}^{\infty} O_{n} \subset I_{R}(\underline{L})$ where $O_{n}$ is an open set, which implies $u \in O_{n}$ for all $n$. Now $w\left(L_{n}{ }^{\prime}\right)$ is an open set for $L_{n} \in \underline{L}$, therefore $u \in w\left(L_{n}{ }^{\prime}\right) \subset O_{n}$ which yields $u \in \bigcap_{n=1}^{\infty} w\left(L_{n}^{\prime}\right) \subset \bigcap_{n=1}^{\infty} O_{n}$. Therefore there exists $u \in Q(\underline{L})$ such that $u \in \stackrel{\cap}{n}=1_{n}^{\infty} w\left(L_{n}^{\prime}\right)$ where the $w\left(L_{n}^{\prime}\right) \downarrow \phi$ and where $\bigcap_{n=1}^{\infty} L_{n} \in \underline{L}$ and $\bigcap_{n=1}^{\infty} w\left(L_{n}^{\prime}\right) \subset I_{R}(\underline{L})-X$, but then $u\left(L_{n}^{\prime}\right)=1$ for all $n$ and $L_{n}^{\prime} \downarrow \phi$ which is a contradiction. Thus $Q(\underline{L}) \subset^{n} \overline{\bar{X}^{\prime}}$ and then by Theorem $4.12, Q(\underline{L})=\bar{X}^{\delta}$.

Using the previous theorem and Theorem 4.2 we have:

COROLLARY 4.15. If $\underline{L}$ is $\delta$-normal separating and disjunctive then $Q(\underline{L})=\bar{X}^{\delta}$ if and only if $Q(\underline{L})=I_{R}(\underline{L}) \cap I_{\sigma}\left(\underline{L}^{\prime}\right)$.

REMARK. We note that $Q(\underline{L})=I_{R}(\underline{L})$ if and only if $C_{b}(\underline{L})=C(\underline{L})$; this situation arises in particular if $C(\underline{L})$ consists only of constant functions. (see below)

5. THE WALLMAN SPACE $I_{R}{ }^{\sigma}(\underline{L})$.

First we note $I_{R}{ }^{\sigma}(\underline{L})$ may be empty. Let $X=\{0,1,2, \ldots\}$ where $\underline{L}$ consists of $\phi$ and all sets of the form $\{n, n+1, \ldots\}$ for all $n$, and $v_{1}, v_{2} \in I_{R}(\underline{L})$. If $v_{1} \neq v_{2}$ then there exists $L_{1}, L_{2} \in \underline{L}$ such that $v_{1}\left(L_{1}\right)=1, \quad V_{2}\left(L_{1}\right)=0, \quad v_{1}\left(L_{2}\right)=0, \quad v_{2}\left(L_{2}\right)=1$ and $L_{1} \cap L_{2}=\phi$. However, this is impossible here as $L_{1} \cap L_{2} \neq \phi$ unless $L_{1}$ or $L_{2}=\phi$. Therefore $I_{R}(\underline{L})=\{u\}$. Now clearly if $L_{n}=\{n, n+1, \ldots\}, L_{n} \in \underline{L}$ and $L_{n} \downarrow \phi$. However, $u\left(L_{n}\right)=1$ for all $n$, therefore $I_{R}{ }^{\sigma}(\underline{L})=\phi$. We also have in this example: $C(\underline{L})=C_{b}(\underline{L})=$ constant functions; $\underline{L}$ is not disjunctive, $\underline{L}$ is not countably paracompact; $\underline{L}$ is not regular; $\underline{L}$ is a $\delta$-lattice.

Now we state a familiar result:

THEOREM 5.1. Let $\underline{L}$ be disjunctive then $\left\langle I_{R}{ }^{\sigma}(\underline{L}), W_{\sigma}(\underline{L})\right\rangle$ is replete.

Next we give facts about $C(\underline{L})$ : we denote by $M_{R}{ }^{I}(\underline{L})$ the set $\left\{u \in M_{R}{ }^{\sigma}(\underline{L})\left|\int_{X}\right| f|d| u \mid<\infty\right.$ for all $f \in C(\underline{L})\}$. Note $I_{R}{ }^{\sigma}(\underline{L}) \subset M_{R}{ }^{I}(\underline{L})$ and we denote by, similar to Varadarajan, $W_{I}$ the topology on $M_{R}^{I}(\underline{L})$. A net $\left\{u_{\alpha}\right\}$ in $M_{R}^{I}(\underline{L})$ converges to $u$ in $M_{R}^{I}(\underline{L})$ with respect to $W_{I}$ if and only if $\int_{X} f d u_{\alpha} \rightarrow \int_{X} f d u$ for all $f \in C(\underline{L})$. The topology $W_{I}$ restricted to $I_{R}{ }^{\sigma}(\underline{L})$ is the Wallman topology. Now using this it is easy to show that $\hat{f}(u)=\int_{X}^{X} f d u, u \in I_{R}{ }^{\sigma}(\underline{L})$ is continuous with respect to the Wallman topology $\tau w_{\sigma}(\underline{L})$ on $I_{R}{ }^{\sigma}(\underline{L})$, i.e., $\hat{f}(u) \in C\left(I_{R}{ }^{\sigma}(\underline{L})\right)=C\left(\tau w_{\sigma}(\underline{L})\right)$. Let $\underline{L}$ be separating, disjunctive and $\delta$-normal throughout and $f \in C(\underline{L})$.

THEOREM 5.2. Let $f \in C(\underline{L})$ then $\hat{f}^{-1}[a, \infty)=Z(\widehat{g})$ where $g=(f-a) \Lambda 0 \in C(\underline{L})$ and similarly $\hat{f}^{-1}(-\infty, a]=Z(\widehat{h})$ where $h \in C(\underline{L})$.

PROOF. Omitted.

THEOREM 5.3. Let $z(\underline{L})$ be the zero lattice of $C(\underline{L})$ then $w_{\sigma}(z(\underline{L}))=z\left(w_{\sigma}(\underline{L})\right)$. 
PROOF. Let $Z \in z(\underline{L}) \subset \underline{L}$. Therefore by a theorem of Alexandroff $Z={ }_{n=1}^{\infty} L_{n}{ }^{\prime}, L_{n} \in \underline{L}$ all $n$. Thus $w_{\sigma}(Z)=\bigcap_{n=1}^{\infty} W_{\sigma}\left(L_{n}\right)^{\prime}$. But $w_{\sigma}(\underline{L})$ is $\delta$-normal therefore by Alexandroff theorem again we get $w_{\sigma}(Z) \in z\left(w_{\sigma}(\underline{L})\right)$. Converse if $w_{\sigma}(L) \in z\left(w_{\sigma}(\underline{L})\right)$, where $L \in \underline{L}$ then $w_{\sigma}(L)=\bigcap_{n=1}^{\infty} w_{\sigma}\left(L_{n}{ }^{\prime}\right)=w_{\sigma}\left(\bigcap_{n=1}^{\infty} L_{n}{ }^{\prime}\right)$ and since $\underline{L}$ is disjunctive, $L=\bigcap_{n=1}^{\infty} L_{n}^{\prime} \in z(\underline{L})$ again by Alexandroff's result and the proof is completed.

We have seen that if $f \in C(\underline{L})$ then $\hat{f} \in C\left(\tau w_{\sigma}(\underline{L})\right)$, i.e., it is continuous with respect to Wallman topology on $I_{R}{ }^{\sigma}(\underline{L})$. However we can do better.

THEOREM 5.4. If $f \in C(\underline{L})$ then $\hat{f} \in C\left(w_{\sigma}(\underline{L})\right.$ ) (where $\hat{f}(u)=\int_{X} f d u$ for all $u \in I_{R}{ }^{\sigma}(\underline{L})$ ).

PROOF. We must show $\hat{f}^{-1}(E) \in w_{\sigma}(\underline{L})$ for any closed set $\underset{E}{X} \subset R$. It will suffice to show this for $E=[a, b] \subset R$. Now $[a, b]=(-\infty, b] \cap[a, \infty)$ so $\hat{f}^{-1}[a, b]=\hat{f}^{-1}[(-\infty, b] \cap[a, \infty)]=\hat{f}^{-1}(-\infty, b]$ $\cap \widehat{f}^{-1}[a, \infty)=Z(\hat{h}) \cap Z(\hat{g})$ using Theorem 5.2. Next we note if $g \in C(\underline{L})$ then $Z(\hat{g})=\overline{Z(g)}$ where the closure is taken in the Wallman space $I_{R}{ }^{\sigma}(\underline{L})$ with topology of closed sets $\tau w_{\sigma}(\underline{L})$. Therefore $\hat{f}^{-1}[a, b]=\overline{Z(g)} \cap \overline{Z(h)}$, and $Z(g), Z(h) \in z(\underline{L}) \subset \underline{L} \quad$ sof $\hat{f}^{-1}[a, b]=\overline{Z(g) \cap Z(h)}$ (using $\bar{A} \cap \bar{B}=\overline{A \cap B}$ for $A, B \in \underline{L}))$. In addition $\hat{f}^{-1}[a, b]=\overline{Z\left(g^{2}+h^{2}\right)}$ and $Z\left(g^{2}+h^{2}\right)=Z \in x(\underline{L})$ and $\overline{Z\left(g^{2}+h^{2}\right)}=\bar{Z}=w_{\sigma}(Z)$. Therefore $\hat{f}^{-1}[a, b]=\bar{Z}=w_{\sigma}(Z)$ which implies $\hat{f}^{-1}[a, b] \in w_{\sigma}(z(\underline{L}))$. However using Theorem 5.3 we get $\hat{f}^{-1}[a, b] \in z\left(w_{\sigma}(\underline{L})\right)$. However $z\left(w_{\sigma}(\underline{L})\right) \subset w_{\sigma}(\underline{L})$ therefore $\hat{f}^{-1}[a, b] \in w_{\sigma}(\underline{L})$ which implies $\hat{f} \in C\left(w_{\sigma}(\underline{L})\right)$.

- Now we intend to prove the converse. Suppose that $h \in\left(w_{\sigma}(\underline{L})\right)$ then clearly $\left.h\right|_{X} \in C(\underline{L})$ and let $\left.h\right|_{X}=f \in C(\underline{L})$ then $h=\hat{f}$ since both are continuous with respect to the Wallman topology and they agree on $X$ which is dense in $I_{R}{ }^{\sigma}(\underline{L})$.

Using the above results we have the following:

THEOREM 5.5. The correspondence $f \rightarrow \hat{f}$ is a bijection between $C(\underline{L})$ and $C\left(w_{\sigma}(\underline{L})\right)$; the $w_{\sigma}(\underline{L})$-continuous functions on the Wallman space $I_{R}{ }^{\sigma}(\underline{L})$.

Next let $u \in I_{R}(\underline{L})$, then we define $M^{u}=\left\{f \in C(\underline{L}) \mid u \in \overline{Z(f)}^{I} R^{(\underline{L})}\right\}$. The following facts we list for completeness (proofs can be found for this setting in [8]):

1) If $u_{1}, u_{2} \in I_{R}(\underline{L})$ and if $u_{1} \neq u_{2}$ then $M^{u_{1}} \neq M^{u_{2}}$.

2) $\boldsymbol{M}^{u}$ is a maximal ideal in $C(\underline{L})$.

3) (Generalized Gelfand-Kolmogoroff) If $M$ is a maximal ideal in $C(\underline{L})$ then there exists $u \in I_{R}(\underline{L})$ such that $M=M^{u}$.

Thus there exists a one to one correspondence between elements of $I_{R}(\underline{L})$ and maximal ideals of $C(\underline{L})$.

Now we return to the Wallman space $\left\langle I_{R}{ }^{\sigma}(\underline{L}), \tau w_{\sigma}(\underline{L})\right\rangle$ and give conditions when this topological space is realcompact. We know that for $\underline{L}$ disjunctive $w_{\sigma}(\underline{L})$ is replete; the question we are now concerned with is: when is the lattice $z\left(\tau w_{\sigma}(\underline{L})\right)$ replete? or i.e., when is the Wallman space realcompact?

THEOREM 5.6. Let $\underline{L}$ be $\delta$-normal, separating, disjunctive, and countably paracompact then $Q(\underline{L})=I_{R}{ }^{\sigma}(\underline{L})$ and if $I_{R}{ }^{\sigma}(\underline{L})$ with the Wallman topology is a c.b. space then it is realcompact.

PROOF. $Q(\underline{L})=I_{R}{ }^{\sigma}(\underline{L})$ by Theorem 4.7. Now $\left\langle I_{R}{ }^{\sigma}(\underline{L}), w_{\sigma}(\underline{L})\right\rangle$ is replete from Theorem 5.1. Now $w_{\sigma}(\underline{L}) \subset \tau w_{\sigma}(\underline{L})$ (of course) and consequently $z\left(\tau w_{\sigma}(\underline{L})\right) \subset \tau w_{\sigma}(\underline{L})$. Now $\underline{L} \delta$-normal implies $w_{\sigma}(\underline{L}) \delta$-normal and $\underline{L}$ countably paracompact implies $w_{\sigma}(\underline{L})$ is countably paracompact. Then by Theorem 5.3 of [2] we have $\tau w_{\sigma}(\underline{L})$ is replete. Now by hypothesis $\tau w_{\sigma}(\underline{L})$ is $z\left(\tau w_{\sigma}(\underline{L})\right)$ countably bounded (c.b.). Thus $z\left(\tau w_{\sigma}(\underline{L})\right)$ is replete by Theorem 3.4 of [2]. Hence $\left\langle I_{R}^{\sigma}(\underline{L}), \tau w_{\sigma}(\underline{L})\right\rangle$ is realcompact.

Note. If $\tau w_{\sigma}(\underline{L})$ is $z\left(\tau w_{\sigma}(\underline{L})\right)$ countably paracompact the same conclusion can be drawn. 
We continue to assume that $\underline{L}$ is separating, disjunctive and $\delta$-normal. Let $h \in C\left(I_{R}{ }^{\sigma}(\underline{L})\right)$ or, i.e., $h \in C\left(\tau w_{\sigma}(\underline{L})\right)$ in lattice notation, then $f=\left.h\right|_{X} \in C(\tau \underline{L})$, clearly. If $f \in C(\underline{L})$ then by our earlier work in this section we would have $h=\hat{f} \in C\left(w_{\sigma}(\underline{L})\right)$. This situation arises if $X$ is a Tychonoff topological space and $\underline{L}=z$ lattice of zero sets of continuous functions on $X$ for in this case if $h \in C\left(I_{R}{ }^{\sigma}(z)\right)$ then $\left.h\right|_{X} \in C(\tau z)=C(F)=C(z)$ where $F$ is the lattice of closed sets of $X$. Thus, in this case, $w_{\sigma}(z)=z\left(\tau w_{\sigma}(z)\right)$ and since $w_{\sigma}(z)$ is replete, we have that $I_{R}{ }^{\sigma}(z)$ is realcompact with respect to the Wallman space.

THEOREM 5.7. Let $\underline{L}$ be separating, disjunctive and $\delta$-normal. If $C\left(\tau w_{\sigma}(\underline{L})\right)=C\left(w_{\sigma}(\underline{L})\right)$ then $z\left(w_{\sigma}(\underline{L})\right)=z\left(\tau w_{\sigma}(\underline{L})\right)$ and if $w_{\sigma}(\underline{L})$ is $z\left(w_{\sigma}(\underline{L})\right)$ c.b. or countably paracompact then $I_{R}{ }^{\sigma}(\underline{L})$ with the Wallman topology is realcompact.

PROOF. Since $w_{\sigma}(\underline{L}) \subset \tau w_{\sigma}(\underline{L})$ then $z\left(w_{\sigma}(\underline{L})\right) \subset z\left(\tau w_{\sigma}(\underline{L})\right)$. Now let $Z(f) \in z\left(\tau w_{\sigma}(\underline{L})\right)$ where $f \in C\left(\tau w_{\sigma}(\underline{L})\right)$, but $C\left(\tau w_{\sigma}(\underline{L})\right)=C\left(w_{\sigma}(\underline{L})\right)$. This implies $Z(f) \in z\left(w_{\sigma}(\underline{L})\right)$. Therefore $z\left(\tau w_{\sigma}(\underline{L})\right) \subset z\left(w_{\sigma}(\underline{L})\right)$. Now if $w_{\sigma}(\underline{L})$ is $z\left(w_{\sigma}(\underline{L})\right)$ countably bounded or countably paracompact then since $w_{\sigma}(\underline{L})$ is replete we have using the same argument as in the proof of Theorem 5.6 that $z\left(w_{\sigma}(\underline{L})\right)$ is replete, therefore $z\left(\tau w_{\sigma}(\underline{L})\right)$ is replete.

Finally we extend Theorem 5.7 but first note $z\left(w_{\sigma}(\underline{L})\right) \subset w_{\sigma}(\underline{L}) \subset \tau w_{\sigma}(\underline{L})$ and $z\left(w_{\sigma}(\underline{L})\right)$ $\subset z\left(\tau w_{\sigma}(\underline{L})\right) \subset \tau w_{\sigma}(\underline{L})$.

THEOREM 5.8. Let $\underline{L}$ be separating, disjunctive and $\delta$-normal. If $\underline{L}$ is $z(\underline{L})$ countably bounded (c.b.) or $\underline{L}$ is $z(\underline{L})$-countably paracompact and assume $z\left(\tau w_{\sigma}(\underline{L})\right) \subset \tau z\left(w_{\sigma}(\underline{L})\right)$, then $z\left(\tau w_{\sigma}(\underline{L})\right)$ is replete, i.e., $I_{R}{ }^{\sigma}(\underline{L})$ with the Wallman topology is realcompact.

PROOF. $z\left(w_{\sigma}(\underline{L})\right)$ is complement generated since $z(\underline{L})$ is complement generated. (Use Theorem 5.3) and $z\left(w_{\sigma}(\underline{L})\right) \subset z\left(\tau w_{\sigma}(\underline{L})\right) \subset \tau z\left(w_{\sigma}(\underline{L})\right)$, therefore by Theorem 3.1 part (1) of [2] we have $z\left(\tau w_{\sigma}(\underline{L})\right)$ is replete, as $z\left(w_{\sigma}(\underline{L})\right)$ is replete from the fact $\underline{L}$ is $z(\underline{L})$ countably bounded or $\underline{L}$ is $z(\underline{L})$ countably paracompact.

ACKNOWLEDGEMENT. The author wishes to express his thanks to Jersey City State College for support of this paper through a grant of partial release time from teaching.

\section{REFERENCES}

1. ALEXANDROFF, A.D. Additive set functions in abstract spaces, Mat. Sb. (N.S.) Volume 8 (50) (1940), 307-348; Volume 9 (51) (1941), 563-628; and Volume 13 (55) $\frac{(1943)}{(136-268 .}$

2. BACHMAN, G \& STRATIGOS, P., On general lattice repleteness and completeness, Illinois Journal of Mathematics, Vol. 27, No. 4 (1983), 535-561.

3. CAMACHO, JR., J., Extensions of lattice regular measures with applications, J. Ind. Math. Soc. 54 (1989), 233-244.

4. EID, G., On normal lattices and Wallman spaces, Internat. J. Math. \& Math. Sci., Volume 13, No 1 (1990) 31-38.

5. FROLIK, Z., Prime filters with the C.I.P., Comm. Math. Univ. Carolinae 13 (1972), 553-575.

6. GRASSI, P., On subspaces of replete and measure replete spaces, Canad. Math. Bull. No. 1, $27(1984), 58-64$.

7. STRATIGOS, P., Some topologies on the set of lattice regular measures, Internat. J. Math. \& Math. Sci. (to appear).

8. SULTAN, A., Lattice realcompactifications, Ann. di Mat. Pura ed Aplicata, Series 4, 106 (1975), 293-303. 
9. SZETO, M., On separation of lattices, Internat. J. Math. \& Math. Sci. Volume 14, No 2 (1991), 325-338.

10. VARADARAJAN, V.S., Measures on topological spaces, Amer. Math. Soc. Transl., Series 2, Volume 48 (1965), 161-228.

11. YALLAOUI, E.B., Induced measures on Wallman spaces, Internat. J. Math \& Math. Sci., Volume 13, No. 4 (1990), 783-798. 


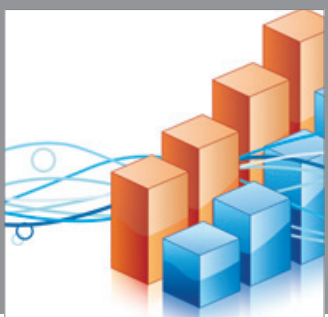

Advances in

Operations Research

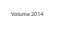

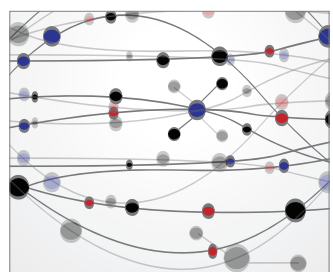

\section{The Scientific} World Journal
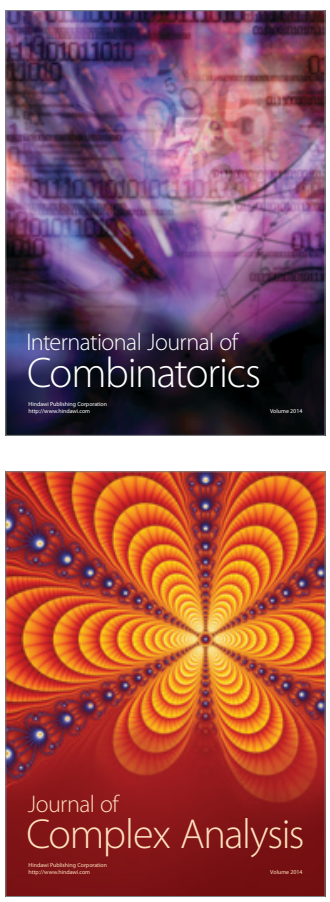

International Journal of

Mathematics and

Mathematical

Sciences
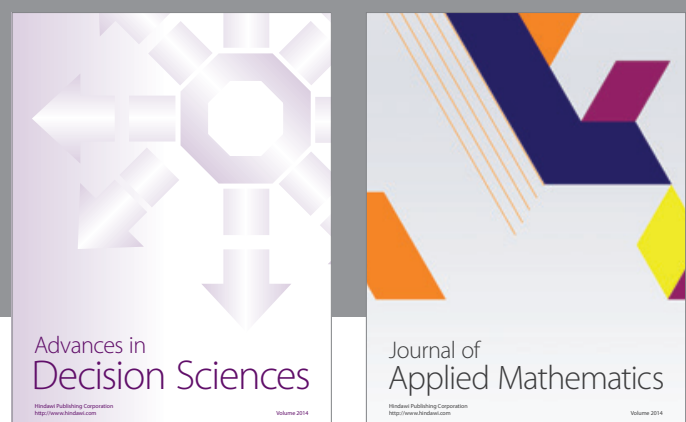

Journal of

Applied Mathematics
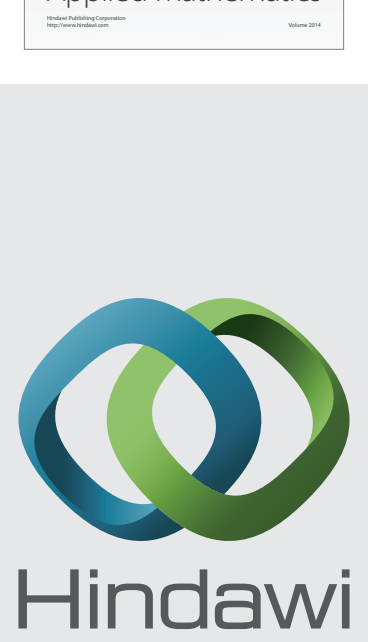

Submit your manuscripts at http://www.hindawi.com
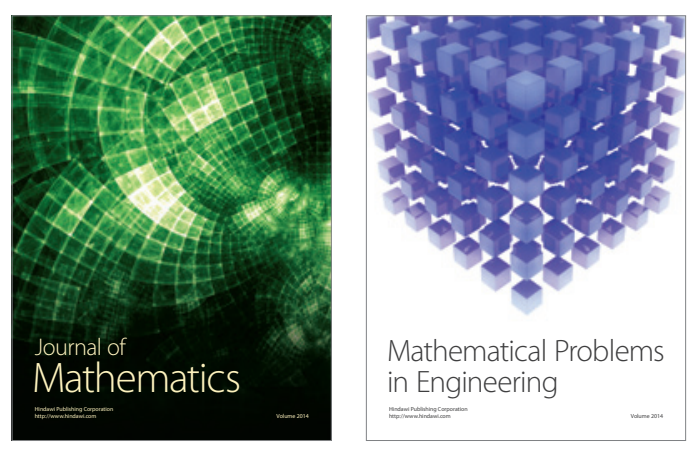

Mathematical Problems in Engineering
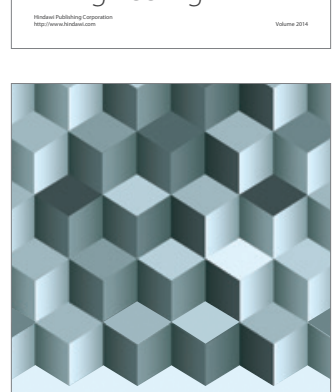

Journal of

Function Spaces
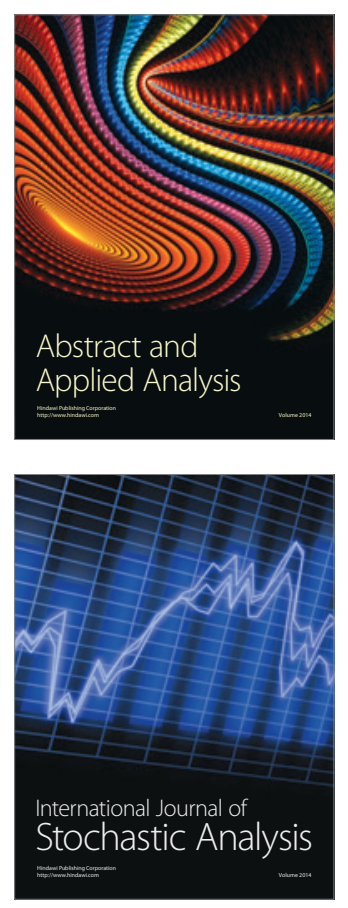

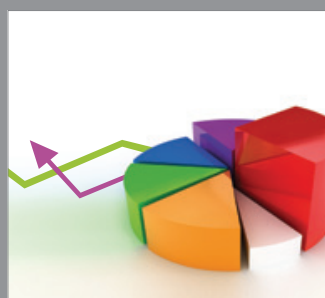

ournal of

Probability and Statistics

Promensencen
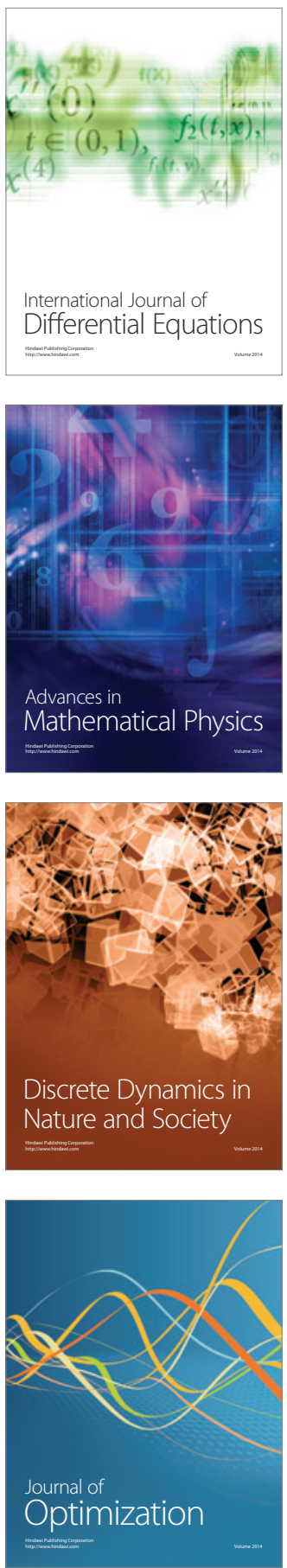